\title{
Universal Scaling Behavior of Anomalous Hall Effect and Anomalous Nernst Effect in Itinerant Ferromagnets
}

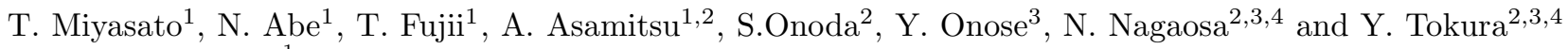 \\ ${ }^{1}$ Cryogenic Research Center, University of Tokyo, Tokyo 113-0032, Japan \\ ${ }^{2}$ Spin Superstructure Project, ERATO, JST, AIST Central 4, Tsukuba 305-8562, Japan \\ ${ }^{3}$ Department of Applied Physics, University of Tokyo, Tokyo 113-8656, Japan \\ ${ }^{4}$ Correlated Electron Research Center(CERC), AIST Central 4, Tsukuba 305-8562, Japan
}

(Dated: June 9, 2018)

\begin{abstract}
Anomalous Hall effect (AHE) and anomalous Nernst effect (ANE) in a variety of ferromagnetic metals including pure metals, oxides, and chalcogenides, are studied to obtain unified understandings of their origins. We show a universal scaling behavior of anomalous Hall conductivity $\sigma_{x y}$ as a function of longitudinal conductivity $\sigma_{x x}$ over five orders of magnitude, which is well explained by a recent theory of the AHE taking into account both the intrinsic and extrinsic contributions. ANE is closely related with $\mathrm{AHE}$ and provides us with further information about the low-temperature electronic state of itinerant ferromagnets. Temperature dependence of transverse Peltier coefficient $\alpha_{x y}$ shows an almost similar behavior among various ferromagnets, and this behavior is in good agreement quantitatively with that expected from the Mott rule.
\end{abstract}

PACS numbers: $72.15 \mathrm{~Eb}, 72.20 \mathrm{~Pa}$

It has been known that Hall resistivity $\rho_{y x}$ in an itinerant ferromagnet has an extra contribution from spontaneous magnetization $M$, which is often expressed empirically by the formula $\rho_{y x}=R_{0} H+4 \pi R_{S} M$, where $R_{0}$ and $R_{S}$ denote ordinary and anomalous Hall coefficient, respectively, and $R_{S}$ is usually a function of the resistivity of materials [1]. The origin of anomalous Hall effect (AHE), however, has long been an intriguing but controversial issue since 1950s. Some of the theories explain AHE from extrinsic origins such as skew scattering $\left(\rho_{y x} \propto \rho_{x x}\right)[2]$ or side-jump $\left(\rho_{y x} \propto \rho_{x x}^{2}\right)[\underline{3}$ mechanisms due to the spin-orbit interaction.

In contrast to these extrinsic mechanisms, several works point out the intrinsic origin of the AHE, which is closely related to the quantal Berry phase on Bloch electrons in solids [4, 5, 6, 7, 8]: the intrinsic part of the anomalous Hall conductivity is given by the sum of the Berry-phase curvature of the Bloch wavefunction over the occupied states, in an analogy to the quantum Hall effect. This Berry-phase scenario of the AHE has recently attracted much interest for its dissipationless and topological nature. Using first-principle band calculations, the intrinsic anomalous Hall conductivity has been calculated for ferromagnetic semiconductors [8, 9], transition metals 10, 11, 12], and oxides 13, 14, in quantitative agreement with experimental results. For example, the AHE in ruthenates $\left(\mathrm{SrRuO}_{3}\right)$ was found to be very sensitive to details of the electronic band structure such as the location of (nearly) crossing points of band dispersions. Such a momentum point acts as a "magnetic monopole" yielding a large Berry-phase curvature and resulting in a resonant enhancement of the anomalous Hall conductivity [13].

Recently, a theory of AHE has been developed taking into account this resonant contribution from the band crossing, where both the topological dissipationless current and dissipative transport current are treated in the presence of the impurity scattering in a unified way 15 . It proposes three scaling regimes for the AHE as a function of the electron lifetime or the resistivity.

In the present paper, we report the anomalous Hall effect in a variety of itinerant ferromagnets at low temperatures, where the magnetization of materials is almost saturated. We have performed AHE measurement on pure metals ( $\mathrm{Fe}, \mathrm{Co}, \mathrm{Ni}$, and $\mathrm{Gd}$ films), oxides $\left(\mathrm{SrRuO}_{3}\right.$ crystal(SRO) 13], $\mathrm{La}_{1-x} \mathrm{Sr}_{x} \mathrm{CoO}_{3}$ crystals(LSCoO) 16|), and chalcogenide-spinel crystals $\left(\mathrm{Cu}_{1-x} \mathrm{Zn}_{x} \mathrm{Cr}_{2} \mathrm{Se}_{4}\right)$ [18]. We have found a universal scaling behavior of the transverse conductivity $\sigma_{x y}$ as a function of longitudinal conductivity $\sigma_{x x}$, which is in good quantitative agreement with a unified theory of the AHE taking into account both intrinsic and extrinsic origins 15. In addition to AHE, we have also examined anomalous Nernst effect (ANE, transverse thermoelectric effect in the presence of spontaneous magnetization), which will provide us with another useful information on the electronic ground state and its relation to the Berry-phase scenario on the AHE.

We used thin films of $\mathrm{Fe}, \mathrm{Co}, \mathrm{Ni}$ and $\mathrm{Gd}$ with the thickness of $1 \mu \mathrm{m}$ and the purity of $99.85 \%, 99.9 \%, 99+\%$, $99.9 \%$, respectively. Single crystals of $\mathrm{La}_{1-x} \mathrm{Sr}_{x} \mathrm{CoO}_{3}$ $(\mathrm{x}=0.17,0.25$ and 0.30$)$ and $\mathrm{SrRuO}_{3}$ were grown by a floating-zone method and a flux method, and whose Curie temperatures are $120 \mathrm{~K}, 225 \mathrm{~K}, 235 \mathrm{~K}$, and $160 \mathrm{~K}$, respectively. The Hall resistivity $\rho_{y x}$ was measured using a Physical Properties Measurement System (Quantum Design Co., Ltd.) together with the longitudinal resistivity $\rho_{x x}$ as a function of magnetic field $(H)$ and temperature $(T)$. The transverse thermopower $\mathrm{Q}_{y x}=E_{y} / \partial_{x} T$ was measured using the same platform by introducing necessary wirings. We apply a temperature gradient $\partial_{x} T$ 


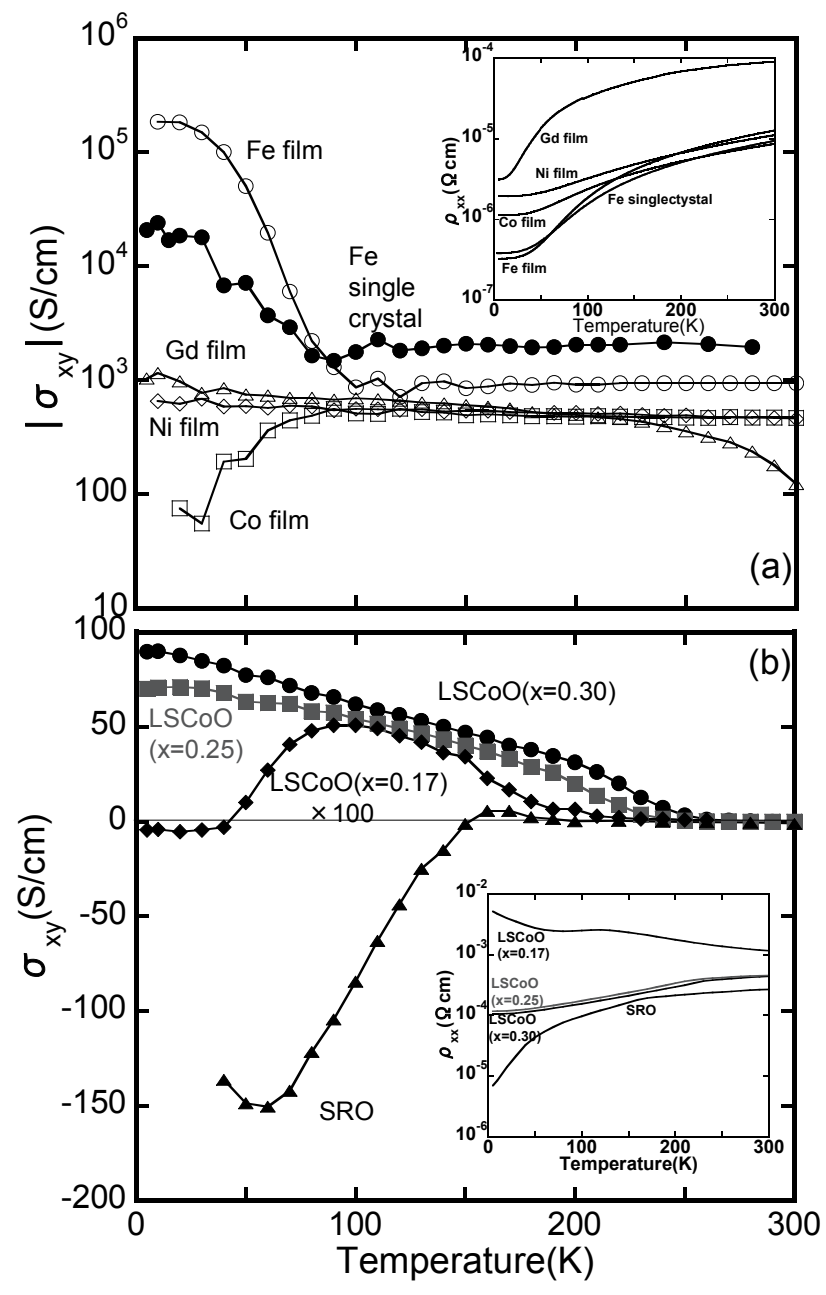

FIG. 1: Temperature dependence of anomalous Hall conductivity $\sigma_{x y}$ for (a) pure metals in logarithmic scale and (b) oxides in linear scale. In top panel, $\sigma_{x y}$ is negative quantity for $\mathrm{Ni}$ and $\mathrm{Gd}$. Temperature dependence of longitudinal resistivity $\rho_{x x}$ is also shown in the inset.

to an electrically isolated sample in a magnetic field and measure the transverse voltage appeared. According to the linear transport theory, we have $\vec{j}=\tilde{\sigma} \vec{E}+\tilde{\alpha}(-\nabla T)$ and $\vec{E}=\tilde{\rho} \vec{j}+\tilde{Q} \nabla T$, where $\vec{j}$ stands for the electric current and $\vec{E}$ for the electric field, and $\tilde{\sigma}, \tilde{\rho}, \tilde{\alpha}$, and $\tilde{Q}$ denote conductivity-, resistivity-, Peltier-, and thermoelectric tensors, respectively. Then we obtain $Q_{x y}=$ $-E_{y} / \partial_{x} T+Q_{x x}\left(\partial_{y} T / \partial_{x} T\right)$ as $\vec{j}=0$. Because we confirmed that the second term is negligibly small compared to the first term, we defined $Q_{x y}=-E_{y} / \partial_{x} T$ in the following. The anomalous contribution in $\rho_{y x}$ and $Q_{x y}$ was determined by extrapolating $\rho_{y x}$ and $\mathrm{Q}_{x y}$ vs. $H$ curves to $H=0$. The transverse conductivity $\sigma_{x y}$ was estimated as $-\rho_{x y} / \rho_{x x}^{2}$ and transverse Peltier coefficient $\alpha_{x y}$ as $\left(Q_{x y}-Q_{x x} \tan \theta_{x y}\right) / \rho_{x x}$, where $\theta_{x y}$ being the Hall angle. The contribution from magnetoresistance or magnetothermopower was carefully removed by subtracting $\rho_{y x}(-H)$ from $\rho_{y x}(H)$ or $Q_{x y}(-H)$ from $Q_{x y}(H)$.
Figure 1 shows the temperature dependence of anomalous Hall conductivity $\sigma_{x y}$ in pure metals(upper panel, (a)) and oxides(bottom panel, (b)). Note that the scale of vertical axis in Fig.1 (a) is logarithmic of $\left|\sigma_{x y}\right|$ while it is linear in Fig.1 (b). All the ferromagnets studied are metallic except $\mathrm{LSCoO}(x=0.17)$ as seen in the inset of Fig.1. In pure metals, the value of $\left|\sigma_{x y}\right|$ is almost constant with $100-1000 \mathrm{~S} / \mathrm{cm}$ below room temperature down to $100 \mathrm{~K}$, and then varies significantly for Fe and Co down to absolute zero. The magnetization $M$ in pure metals is constant below room temperature (not shown). In oxides, the change of $\sigma_{x y}$ is very complicated due to the change of $M$ and even shows the sign change in SRO and $\mathrm{LSCoO}$.

A striking relation among various $\sigma_{x y}$ values becomes apparent if we focus on $\sigma_{x y}$ at a lowest temperature. Figure 2 shows the variation of the absolute value of anomalous Hall conductivity $\left|\sigma_{x y}\right|$ against the longitudinal conductivity $\sigma_{x x}$ over five orders of magnitude in the ground state of itinerant ferromagnets. The data for $\mathrm{Cu}_{1-x} \mathrm{Zn}_{x} \mathrm{Cr}_{2} \mathrm{Se}_{4}$ is included in the figure. For pure metals, all data of $\left|\sigma_{x y}\right|$ below room temperature are also plotted. The variation of $\left|\sigma_{x y}\right|$ can be categorized into three regions: In the intermediate region with $\sigma_{x x}=10^{4}-10^{6} \mathrm{~S} / \mathrm{cm}$, such as in pure metals and $\mathrm{SrRuO}_{3}$, one can see that $\left|\sigma_{x y}\right|$ is nearly constant $(\simeq 1000$ $\mathrm{S} / \mathrm{cm})$, which means that $\rho_{y x} \propto \rho_{x x}^{2}$. Furthermore, this constant value of $\left|\sigma_{x y}\right|$ is consistent with the "resonant" AHE which gives the intrinsic contribution of the order of $e^{2} / h a \sim 10^{3} \mathrm{~S} / \mathrm{cm}$, with $a$ being a lattice constant [15]. The contributions from the extrinsic mechanisms, i.e., skew-scattering and side-jump, are found to be much smaller than $e^{2} / h a$ in this region. Therefore, we can regard the $\sigma_{x y}$ in the plateau region as the dominantly intrinsic contribution.

In the extremely clean case with $\sigma_{x x} \simeq 10^{6} \mathrm{~S} / \mathrm{cm}$, such as in Fe and Co at low temperatures, the behavior of $\left|\sigma_{x y}\right|$ seems to depend on materials. According to the classical Boltzmann transport theory, impurity scattering gives rise to anomalous Hall conductivity through the skewness or the side jump, and the skew-scattering contribution to AHE diverges in the clean limit as $\sigma_{x y} \propto \sigma_{x x}$. Although the experimental results show a slight deviation from the theoretical prediction, the qualitative change in $\sigma_{x y}$ from the intrinsic region is obvious.

Finally in the dirty limit with $\sigma_{x x}<10^{4} \mathrm{~S} / \mathrm{cm}$, such as in $\mathrm{Cu}_{1-x} \mathrm{Zn}_{x} \mathrm{Cr}_{2} \mathrm{Se}_{4}$ and $\mathrm{La}_{1-x} \mathrm{Sr}_{x} \mathrm{CoO}_{3}$, the intrinsic contribution to AHE is suppressed by the damping effect due to impurities, and the change in anomalous Hall conductivity is well described by $\sigma_{x y} \propto \sigma_{x x}^{1.6}$ experimentally. This exponent is also expected in the "insulator" regime of quantum-Hall systems [19].

The universal scaling behavior above is well explained by a unified theory of the AHE taking into account both intrinsic and extrinsic origins 15]: Three scaling regimes have been found for a generic two-dimensional model 


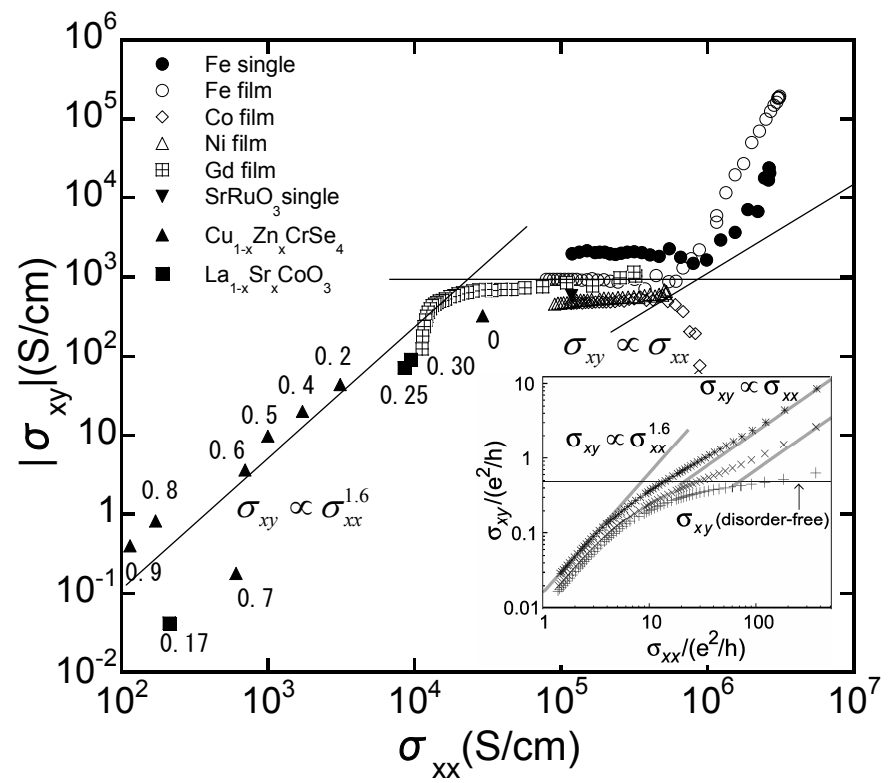

FIG. 2: Absolute value of anomalous Hall conductivity $\left|\sigma_{x y}\right|$ as a function of longitudinal conductivity $\sigma_{x x}$ in pure metals (Fe, Ni, Co, and Gd), oxides $\left(\mathrm{SrRuO}_{3}\right.$ and $\left.\mathrm{La}_{1-x} \mathrm{Sr}_{x} \mathrm{CoO}_{3}\right)$, and chalcogenide spinels $\left(\mathrm{Cu}_{1-x} \mathrm{Zn}_{x} \mathrm{Cr}_{2} \mathrm{Se}_{4}\right)$ at low temperatures. Three lines are $\sigma_{x y} \propto \sigma_{x x}^{1.6}, \sigma_{x y}=$ const., and $\sigma_{x y} \propto \sigma_{x x}$ for dirty, intermediate, and clean regimes, respectively. The inset shows theoretical results obtained from the same analysis as in Fig. 4 of Ref.[15] but for $E_{F}=0.9 ; 2 m v=0.02(+)$, $0.2(\times)$, and $0.6(*)$. Here, $m$ is the effective mass and $v$ is the strength of the $\delta$-functional impurity potential.

containing the resonant enhancement of $\sigma_{x y}$ due to an anti-crossing of band dispersions and the impurity scattering. In the extremely clean case where the relaxation rate $\tau^{-1}$ is smaller than the band energy splitting given by the spin-orbit interaction energy $\varepsilon_{s o}$, the extrinsic skew-scattering contribution gives the leading contribution, yielding the scaling $\sigma_{x y} \propto \sigma_{x x}$. If the Fermi level is located around the anti-crossing of band dispersions, a crossover to the intrinsic regime occurs around $\tau^{-1} \sim \varepsilon_{s o}$, with the resonant enhancement $\sigma_{x y} \sim e^{2} / h$ and the scaling $\sigma_{x y}=$ constant. For the hopping-conduction regime with $\tau^{-1}>E_{F}$ with the Fermi Energy $E_{F}$, there occurs another scaling $\sigma_{x y} \propto \sigma_{x x}^{1.6}$. The present experimental results on the crossover in $\sigma_{x y}$ among clean, intermediate, and dirty cases is thus well reproduced by this theory (see the inset of Fig.2).

Now we move on to the anomalous Nernst effect. The transverse Peltier coefficient $\alpha_{x y}$ is given by the Mott rule,

$$
\alpha_{x y}=\left(\frac{\pi^{2} k_{B}^{2}}{3 e}\right) T \frac{d}{d \epsilon}\left[\sigma_{x y}(\epsilon)\right]_{\mu},
$$

where $k_{B}$ is the Boltzmann constant, $e$ the elementary charge, and $\mu$ the chemical potential [17. In Fig.3, we show $\alpha_{x y}$ and $M$ simultaneously in $\mathrm{La}_{1-x} \mathrm{Sr}_{x} \mathrm{CoO}_{3}(\mathrm{x}=0.3,0.25,0.17)$ and $\mathrm{SrRuO}_{3}$. All the
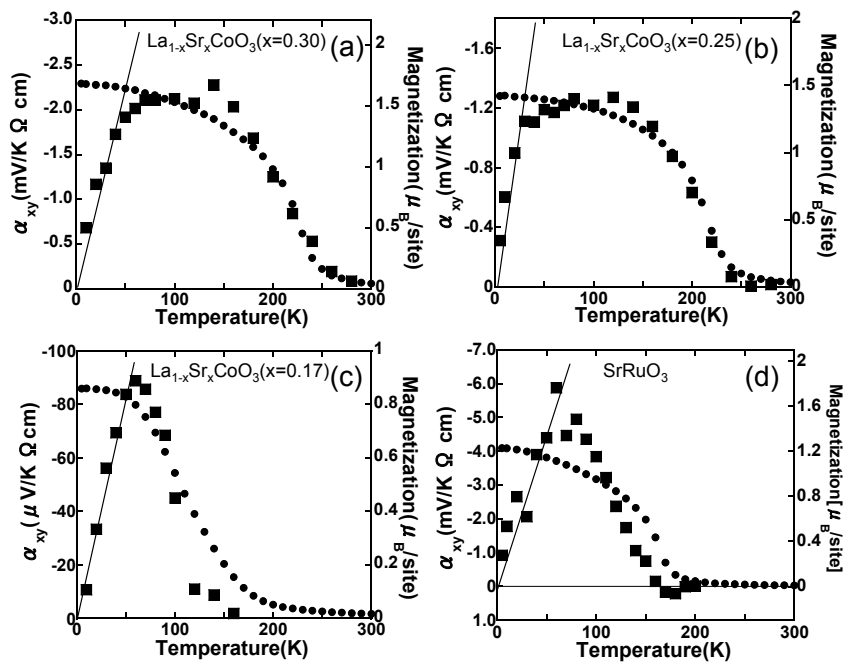

FIG. 3: Temperature dependence of anomalous transverse Peltier coefficient $\alpha_{x y}$ (squares) and magnetization $M$ (circles) in (a) $\mathrm{La}_{1-x} \mathrm{Sr}_{x} \mathrm{CoO}_{3}(x=0.3)$, (b) $\mathrm{La}_{1-x} \mathrm{Sr}_{x} \mathrm{CoO}_{3}$ $(x=0.25)$, (c) $\mathrm{La}_{1-x} \mathrm{Sr}_{x} \mathrm{CoO}_{3}(x=0.17)$, and (d) $\mathrm{SrRuO}_{3}$. The straight line at low temperatures represents $T$-linear variation of $\alpha_{x y}$.

materials (not shown for pure metals) seem to show qualitatively very similar temperature dependence that $\alpha_{x y}$ starts to increase just below $T_{C}$, being almost proportional to $M$, then decreases at low temperatures linearly with $T$, and finally vanishes toward absolute zero. These behaviors are well understood using the above formula: $\alpha_{x y}$ just below $T_{C}$ is subject to the factor $\left(d \sigma_{x y} / d \epsilon\right)_{\mu}$, where the modification of the band structures at the Fermi level takes place due to the ferromagnetic transition. After the saturation of $M, T$-linear term becomes dominant in the change of $\alpha_{x y}$.

In order to confirm the validity of Eq.(1) further, we performed a quantitative analysis on $\alpha_{x y}$ in $\operatorname{LSCoO}(\mathrm{x}=0.3-0.18)$. The equation is rewritten to

$$
\frac{\alpha_{x y}}{T}=\frac{\gamma}{e} \frac{d}{d n}\left[\sigma_{x y}(\epsilon)\right]_{\mu},
$$

with the electronic heat-capacity coefficient $\gamma$ by using the transformation $\frac{d}{d \epsilon}\left[\sigma_{x y}(\epsilon)\right]_{\mu}=\frac{d n}{d \epsilon} \frac{d}{d n}\left[\sigma_{x y}(\epsilon)\right]_{\mu}$. We obtained $\gamma$ from heat-capacity measurement and the carrier density $n$ from the ordinary Hall effect at $300 \mathrm{~K}$ which is far above $T_{C}$. These values are shown in Table \ Because the composition $x$ is nominal, we employed two samples with $x=0.18$ showing different values of physical quantities [20]. The left-hand and right-hand sides (LHS and RHS) of Eq.(2) were estimated independently using these values in the center of Table \ replacing the differential $d \sigma_{x y} / d n$ with the difference $\Delta \sigma_{x y} / \Delta n$, and the relation of both sides was summarized in Fig.4. The proportionality between these two quantities is obvious, and the slope is about 0.85 and slightly different from 1. Although we do not understand the reason for this 
TABLE I: Experimental data of $\alpha_{x y} / T, \sigma_{x y}$ at a lowest temperature, carrier density $n$ derived from the ordinary Hall effect at room temperature, and electronic heat-capacity coefficient $\gamma$ in $\mathrm{La}_{1-x} \mathrm{Sr}_{x} \mathrm{CoO}_{3}$. The left-hand side (LHS) in Eq.(2) is the average of two successive $\alpha_{x y} / T$ and the right-hand side (RHS) is estimated using the average of $\gamma$ and the difference $\Delta \sigma_{x y} / \Delta n$.

\begin{tabular}{|c|c|c|c|c|c|c|}
\hline$x$ & $\alpha_{x y} / T\left(\mu \mathrm{V} / \mathrm{K}^{2} \Omega \mathrm{cm}\right)$ & LHS & RHS & $\sigma_{x y}(\mathrm{~S} / \mathrm{cm})$ & $n\left(10^{23} / \mathrm{mol}\right)$ & $\gamma\left(\mathrm{mJ} / \mathrm{mol} \mathrm{K}^{2}\right)$ \\
\hline 0.30 & -437 & -285 & -339 & 90.3 & 1.43 & 49.1 \\
\hline 0.26 & -132 & -142 & -170 & 34.2 & 1.89 & 39.5 \\
\hline 0.22 & -153 & -85.9 & -103 & 23.8 & 2.04 & 41.1 \\
\hline $0.18-2$ & -19.2 & -27.8 & -8.77 & 1.10 & 2.86 & 19.0 \\
\hline ก 101 & $0<n$ & & & -0.002 & 3.27 & 32.4 \\
\hline
\end{tabular}

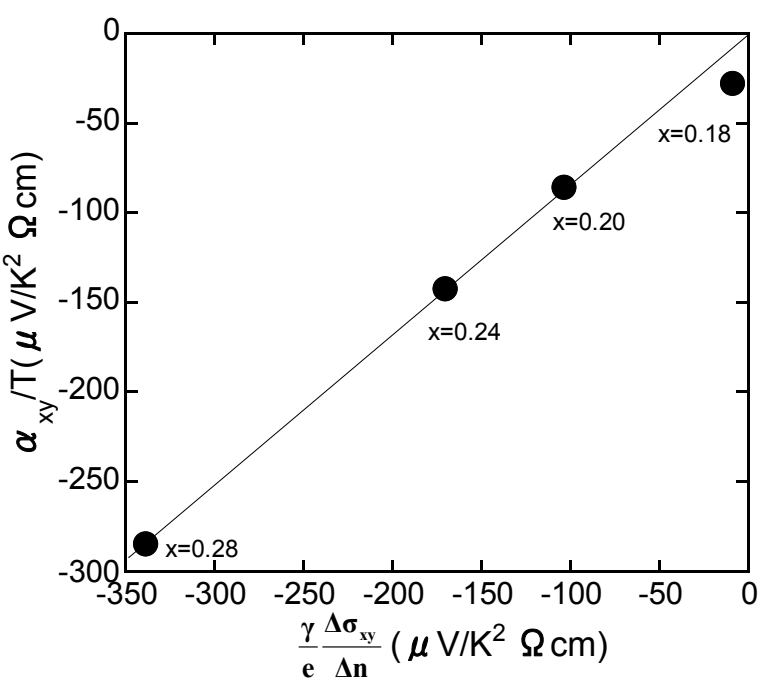

FIG. 4: The relation between the left-hand and right-hand sides of Eq.(2) derived independently using the experimental data in Table $\llbracket$ for $\mathrm{La}_{1-x} \mathrm{Sr}_{x} \mathrm{CoO}_{3}$. The line has a gradient of about 0.85 .

slight discrepancy yet, $\alpha_{x y}$ obeys Eq.(2) quantitatively, and hence the thermoelectric Hall transport property is understandable, at least in the dirty case, in terms of the Mott rule. This may suggest that the Berry-phase contribution to the thermoelectric Hall transport phenomena is dominant mainly through $\sigma_{x y}$.

In summary, we have investigated the anomalous Hall effect and anomalous Nernst effect in various ferromagnetic metals, such as $\mathrm{Fe}, \mathrm{Co}, \mathrm{Ni}, \mathrm{Gd}, \mathrm{La}_{1-x} \mathrm{Sr}_{x} \mathrm{CoO}_{3}$, $\mathrm{SrRuO}_{3}$, and $\mathrm{Cu}_{1-x} \mathrm{Zn}_{x} \mathrm{Cr}_{2} \mathrm{Se}_{4}$. The anomalous Hall conductivity $\sigma_{x y}$ in the ground state shows a universal scaling behavior against the longitudinal conductivity $\sigma_{x x}$, being independent of materials. This scaling relation can be well understood by a recent theory taking into account both intrinsic and extrinsic origin of the AHE. We have also shown that the relation between the anomalous Nernst effect and the anomalous Hall effect can be explained quantitatively by the Mott rule.

This work was partly supported by the Grant-in-
Aid for Scientific Research (Nos.15104006, 16076205, 17105002, and 17038007) from the Ministry of Education, Culture, Sports, Science and Technology, Japan.

[1] C. L. Chien and C. R. Westgate, The Hall Effect and Its Applications (Plenum, New York, 1979).

[2] J. Smit, Physica (Amsterdam) 21, 877 (1955); 24, 39(1958)

[3] L. Berger, Phys. Rev. B 2, 4559(1970)

[4] R. Karplus and J. M. Luttinger, Phys. Rev. 95, 1154(1954); J. M. Luttinger, Phys. Rev. 112, 739(1958).

[5] J. Ye, Y. B. Kim, A. J. Millis, B. I. Shraiman, P. Majumdar, and Z.Tesanovic, Phys. Rev. Lett. 83,3737(1999)

[6] M. Onoda and N. Nagaosa, J. Phys. Soc. Jpn. 71, 19(2002); Y. Taguchi, Y. Ohara, H. Yoshizawa, N. Nagaosa, and Y. Tokura, Science 291, 2573(2001)

[7] Y. Lyanda-Geller, S. H. Chun, M. B. Salamon, P. M. Goldbart, P. D. Han, Y. Tomioka, A. Asamitsu, and Y. Tokura, Phys. Rev. B 63, 184426(2001)

[8] T. Jungwirth, Q. Niu, and A. H. MacDonald, Phys. Rev. Lett. 88, 207208(2002)

[9] C. Zeng, Y. Yao, Q. Niu, and H. H. Weitering Phys. Rev. Lett. 96, 037204(2006)

[10] J. G.Yao et al., Phys. Rev. Lett. 92, 016602(2004)

[11] S. A. Baily and M. B. Salamon, Phys. Rev. B 71, 104407(2005)

[12] J. Kotzler and W. Gil, Phys. Rev. B 72, 060412(2005)

[13] Z. Fang et al., Science 302, 92(2003); R. Mathieu et al., Phys. Rev. Lett. 93, 016602(2004)

[14] L. M. Wang, Phys. Rev. Lett. 96, 077203(2006)

[15] S. Onoda, N. Sugimoto, and N. Nagaosa, Phys. Rev. Lett. 97, 126602(2006)

[16] Y. Onose and Y. Tokura, Phys. Rev. B 73, 174421(2006)

[17] E. H. Sondheimer, Proc. R. Soc. London 193, 484(1948); L. Smrčka and P. Středa, J. Phys. C 10, 2153 (1977).

[18] W.-L. Lee, S. Watauchi, V. L. Miller, R. J. Cava, and N. P. Ong, Science 303, 1647 (2004).

[19] L. P. Pryadko and A. Auerbach, Phys. Rev. Lett. 82, 1253 (2004).

[20] This doping level is close to the metal-insulator boundary in $\mathrm{La}_{1-x} \mathrm{Sr}_{x} \mathrm{CoO}_{3}[16$, and hence the propeties are quite sensitive to slight off-stoichiometry. 